\title{
L'ARTICOLAZIONE DEL POLITICO E DEL RELIGIOSO NELLE DEMOCRAZIE CONTEMPORANEE. UN DIALOGO CON LUCA DIOTALLEVI
}

\author{
Philippe Portier
}

La sociologia politica del religioso ha, nel corso di questi ultimi anni, sviluppato numerose e importanti ricerche sul modo di regolare la credenza nei paesi occidentali. Il testo molto bello di Luca Diotallevi si colloca in questo campo di studi, con l'obiettivo di ripensare su nuove basi le tesi solitamente condivise su questa materia.

Il suo punto di partenza consiste nell'analisi dei tratti principali della produzione scientifica per quanto riguarda la materia in questione. Per il sociologo italiano, tale analisi si articola globalmente intorno a due punti chiave. Segnala, da una parte, un fenomeno di convergenza: fissati ieri in combinazioni singolari, prodotti essi stessi di processi diversificati di nation building, $\mathrm{i}$ sistemi di secolarità avrebbero conosciuto, nel corso di questi ultimi decenni, un ravvicinamento dei loro modelli di organizzazione: la maggior parte ha adottato un sistema di "separatismo moderato". La produzione scientifica ammette, d'altra parte, un fenomeno di resistenza. Due grandi paesi, gli Stati Uniti e la Francia, sono rimasti, per quanto li riguarda, dipendenti dal loro modello originario di "separatismo radicale", proibendo, secondo l'assioma stabilito al momento della loro entrata nelle modernità, qualsiasi cooperazione tra istituzione politica e istituzione religiosa.

Orbene, nessuna di queste due conclusioni conviene a Luca Diotallevi. Il concetto di "separatismo moderato" aggrega regimi di fatto molto differenti, i quali non sviluppano lo stesso tipo di cooperazione tra lo Stato e le Chiese. Accade lo stesso per quanto riguarda il concetto di "separazione radicale", che assimila erroneamente, nel momento in cui non regolano allo stesso modo il rapporto tra privato e pubblico, il regime francese e il regime americano di secolarità. In fondo le critiche convergono: il sociologo italiano rimprovera ai modelli di analisi attualmente in vigore il loro carattere generale, e dunque riduttore: non prendono in considerazione, spiega l'autore, la pluralità, nonostante il processo di globalizzazione, delle esperienze contemporanee della gestione pubblica del credere.

La critica richiede un programma. Luca Diotallevi si dedica a ripercorrere i vari modelli nazionali per coglierli nella loro idiosincrasia. Il suo testo si sofferma in particolare sul caso americano e sul caso francese: intende mostrare che il "separatismo radicale" tra lo Stato e le forze religiose è negli Stati Uniti molto meno rigido che in Francia. Sostenuto da una ricca argomentazione, la tesi risulta del tutto convincente. Tuttavia lascia sussistere alcuni punti deboli, 
che riguardano in particolare la genesi e la plasticità dei modi nazionali di articolazione della relazione Chiese/Stato.

\section{Zone di convergenza}

La zona occidentale del mondo è caratterizzata da quello che Alfred Stepan chiama la "twin toleration" "legata essa stessa al processo di secolarizzazione (definito qui come un processo di perdita di pertinenza sociale degli enunciati di natura religiosa). Da un lato, l'istituzione politica, ormai dissociata dalla volontà di Dio, non interviene più nell'ordine della salvezza: lascia ciascuno alle proprie scelte, e anche alla libertà di non credere. Dall'altro, l'istituzione religiosa, se interviene nella sfera del dibattito pubblico, non determina più la legge collettiva: è solo una voce tra le altre, altrettanto legittima per fondare la decisione politica.

Luca Diotallevi propone, in primo luogo, un'analisi del modo di generazione di questa secolarizzazione giuridica. Il suo schema che deve molto ai lavori di Niklas Luhmann si fonda sul paradigma della differenziazione sociale. Ne conosciamo il principio: ogni gruppo umano, per ridurre la "complessità del mondo, organizza la sua esistenza definendo sfere di attività, tutte orientate da un sistema assiologico (sinn), e separate le une dalle altre da frontiere mutevoli. Ebbene, fa notare il sociologo italiano, questa organizzazione si è evoluta nel corso della storia. L'epoca premoderna ci mette di fronte ad una differenziazione unificata: le funzioni politiche, economiche, scientifiche, artistiche della società si trovano collocate sotto la tutela della norma religiosa. Hermann Tyrell descrive così l'articolazione religione/politica legata a questo modello: "Con la formula del "coge intrare" (ou "compelle intrare"), forgiata da Sant'Agostino, detta altrimenti l'invito, indirizzata allo Stato, di imporre, nel caso fosse necessario, con la costrizione (con mezzi specificamente di Stato) l'appartenenza dei soggetti alla Chiesa, "gruppo politico" e "gruppo ierocratico" finiscono per sovrapporsi, a "coincidere", come dice Weber, per quanto riguarda la loro portata sociale e la loro sfera di dominazione". ${ }^{2}$

L'epoca moderna si costruisce al contrario su una differenziazione pluralista. I sottosistemi diventano autonomi in confronto alla funzione religiosa, e definiscono il proprio "mezzo di

1Alfred Stepan, "The World Religious Systems and Democracy : Crafting the Twin Tolerations », Journal of Democracy, 11, October 2000, 37-57.

2 Hartmann Tyrell, "Religion und Politik. Max Weber und Emile Durkheim », in A. Bienfait, (éd.), Religionen verstehen, Wiesbaden, Verlag für Sozialwissenschaften, 2011, p. 61, 


\section{L'ARTICOLAZIONE DEL POLITICO E DEL RELIGIOSO NELLE DEMOCRAZIE CONTEMPORANEE. UN DIALOGO CON LUCA DIOTALLEVI 3}

comunicazione". Così accade per la sfera politica: questa afferma ormai una sovranità, che la conduce a rifiutare principi normativi che siano diversi da quelli che dà a se stessa. Per Luca Diotallevi, la libertà religiosa del soggetto è correlata con l'autonomia giuridica del potere. È perché le società si costruiscono ormai indipendentemente da una norma inglobante che ciascuno può decidere delle proprie scelte, compreso nella sfera spirituale, senza trovarsi costretto da un potere politico sottomesso all'ordine religioso: “ la differenziazione funzionale produce i diritti”, spiega l'autore. Certamente relazioni istituzionalizzate possono mantenersi tra le due istanze, come è successo nella maggior parte dei paesi europei. Esse non sono più della stessa natura che nella società premoderna, perché la legge politica non dipende più dalla legge canonica.

$\mathrm{Ci}$ ritroviamo d'accordo in questo schema analitico. Per quanto riguarda la relazione di consecuzione, postulata da Diotallevi, tra la sovranità politica e la libertà religiosa, forse bisogna aggiungere questo caveat: la sovranità politica è una condizione necessaria della libertà religiosa; questa non è una condizione sufficiente. Claude Lefort et Emilio Gentile hanno mostrato, nei loro rispettivi lavori sul comunismo e il fascismo, che il politico poteva, anche in regime di modernità, trasformarsi in "religione", e abolire, di fatto, la possibilità della libertà di coscienza. La teoria dei sistemi sociali non può, nella fattispecie, fare a meno della teoria dei regimi politici: affinché la libertà sia protetta, bisogna che, con tutta una serie di dispositivi tecnici, il sistema giuridico metta il potere politico a distanza da qualsiasi concezione particolare del bene (il principio di neutralità) accettando, nell'ordine che regge, qualsiasi espressione religiosa e di convinzione, a condizione che non danneggino altre persone (principio di pluralità).

Il sociologo italiano propone, in secondo luogo, un'analisi del modo di diversificazione della secolarità giuridica. La modernità non ha generato un modello unico di separazione del politico e del religioso: conosce "diverse varianti della differenzazione istituzionale". Il testo cita il modello inglese; fa riferimento anche ai sistemi scandinavi di confessionalismo. Ma si sofferma soprattutto sul caso americano e francese. Numerosi autori li inglobano volentieri, l'abbiamo detto, nella stessa categoria di "separatismo stretto". Una sociologa francese scriveva recentemente in questo senso: "La Francia e gli Stati Uniti appaiono come i campioni della istituzionalizzazione della laicità (...) forti di un inquadramento laico istituito in maniera precoce, durevole e saldo." 3 . Luca Diotallevi

3

Camille Froidevaux, « Religion et politique dans les sociétés sécularisées », Critique internationale, № 44, JuilletSeptembre 2009, p.15. 
difende un'altra tesi. Pensa, per quanto lo riguarda, che i due paesi, pur utilizzando la stessa parola, non coltivano, di fatto, la stessa pratica di separatismo. In America il separatismo è flessibile, e definisce un regime di "libertà religiosa": oltre al fatto che la libertà di religione fonda, qui, la liberta di coscienza", lo "spazio pubblico" è aperto all'intervento dei "discorsi e degli "attori" religiosi. La Francia presenta tratti diametralmente opposti: ha fatto della libertà di religione una conseguenza della libertà di coscienza, e, a partire dalla legge di separazione del 9 dicembre 1905, ha chiuso la sfera dello Stato a qualsiasi intromissione della potenza ecclesiastica. La Francia descrive "un regime di laicità".

La descrizione continua con uno schema esplicativo. Alla maniera di un Pierre Birnbaum in Francia o di un Mathias Koening in Germania, Luca Diotallevi colloca la variabile dello Stato al primo piano della sua dimostrazione. Negli Stati Uniti, spiega, si tratta di una Stateless Society. Lo Stato è debole. Non ha nessuna vocazione ad essere, secondo l'espressione di Emile Durkheim, il "rettore" della società. Si accontenta, con il sostegno di un sistema giuridico indipendente dal sistema politico, di articolarne gli innumerevoli pluralismi, senza volerli ridurre all'unità. Questo schema è tanto più radicato in quanto il modello di organizzazione del politico è qui di tipo federale. In questa configurazione, le frontiere del politico diventano necessariamente porose, di conseguenza, suscettibili a potersi aprire, più facilmente che altrove, al religioso. La Francia ci confronta, al contrario, soprattutto a partire della Terza Repubblica (1880) ad un modello di Stato forte (Strong State). Il potere si colloca qui al di sopra della società. Alla maniera della Chiesa cattolica da cui riprende mimeticamente i tratti, convinto di essere l'unico depositario della ragione universale, intende costruire dall'alto, sul fondamento della sua legge e della sua scuola, un mondo della generalità pubblica, dove ciascuno potrà domani, dissociandosi dagli arcaismi religiosi, accedere alla "maggior età". Non si tratta chiaramente, salvo nel caso di alcuni repubblicani (minoritari), di sradicare il religioso dall'orizzonte umano. S'intende per lo meno contenerlo nel solo spazio privato dei soggetti.

Si potrebbe illustrare la tesi di Luca Diotallevi con due esempi. Il primo riguarda la questione di indossare segni religiosi nella scuola pubblica. La Francia lo ha proibito con la legge del 15 marzo 2004, giustificandolo con la necessità di contribuire all'emancipazione delle giovani riguardo agli obblighi confessionali, e per evitare che la scuola della Repubblica diventasse uno

4Visto che la religione è ciò che si vive in coscienza. Voir R. Forst, Toleranz im Konflikt : Geschichte, Gehalt und Gegenwart eines umstrittenen Begriffs, Francfort-sur-le-Main, Suhrkamp, 2003. 


\section{L'ARTICOLAZIONE DEL POLITICO E DEL RELIGIOSO NELLE DEMOCRAZIE CONTEMPORANEE. UN DIALOGO CON LUCA DIOTALLEVI 5}

spazio diviso in comunità giustapposte. Gli Stati Uniti, nell'insieme, non hanno capito questa legge, legata, infatti ad un tipo di universalismo di Stato che non praticano. Hanno, per conto loro, in nome della loro "tolleranza di principio", ammesso, senza nessuna difficoltà, la possibilità di indossare segni religiosi a scuola. Lo Stato qui non desidera produrre unità. Il suo ruolo è piuttosto quello di articolare le differenze. Il secondo esempio riguarda la questione del modo di costituzione della religione civile. In Francia, infatti, i legami tra la religione civile nazionale laica e il cristianesimo, in particolare la Chiesa cattolica, sono rotti a partire dagli anni 1880. È perché il cattolicesimo è da allora bandito dallo spazio pubblico, che lo Stato-nazione è condotto, nella rappresentazione che dà di se stesso, a rinunciare a qualsiasi utilizzo del repertorio tradizionale dei simboli cristiani. Niente di tutto questo in America. Senza legarsi a denominazioni particolari, la religione civile è qui largamente debitrice di una spiritualità deista, nella quale ciascuno, senza la necessità di conflitti con la propria Church religion, può riconoscersi.

\section{Punti di discussione}

Il testo di Luca Diotallevi offre un modello analitico di prima importanza. Tuttavia può suscitare discussioni. Probabilmente, questo è dovuto alla sua qualità: così come in Luhmann, la modellizzazione si accompagna di un certo deficit di storicità, dovuto probabilmente, come indicava Jürgen Habermas nella sua critica del sistemismo, alla sottovalutazione della dinamica della soggettività. Vorremo qui provare a restituire della diacronia nella sincronia dell'argomento, interrogando poi, come l'abbiamo detto prima, la genesi e il divenire dei sistemi occidentali del modo di regolare la credenza.

La prima domanda riguarda dunque la costituzione dei modelli di relazioni Chiese/Stato. Non si capisce bene nel discorso di Diotallevi perché i Francesi avrebbero difeso l'egemonia del politico e gli Americani quello dell'apertura al religioso. La risposta è collegata con il processo di costruzione dello Stato-nazione. Due elementi chiave distinguono i due paesi. In primis, la loro traiettoria politica. In Francia, fin dall'epoca barocca, lo Stato si colloca già, per ridurre le rotture nate dalle guerre di religione, al di sopra delle diversità delle società locali. Questi (lo Stato) ha istallato, nelle province, degli amministratori collegati al centro parigino e imponendo così alle periferie , contro le tradizioni locali stabilite, la legge del monarca. Per quanto riguarda la questione 
della gestione della coscienza pubblica, tramite le regole del gallicanismo, questi sottomette la Chiesa cattolica al suo controllo, pur difendendo, è vero, la sua egemonia formale. Qui, la "dinamica dell'Occidente" descritta da Norbert Elias, gioca a pieno: confrontato a una resistenza forte da parte delle unità locali, il potere governativo si salda intorno ad un governo centrale potente. Questa è la premessa di un pensiero dell'unità dello Stato, che la Rivoluzione del 1789, l'Impero di Napoleone, e presto la Repubblica rinforzerà sempre più. Il processo di nation-building non è stato lo stesso negli Stati Uniti, dove lo Stato si è edificato par remontée confédérale (in un primo tempo) senza configurazione autoritaria del legame politico.

Le strutture religiose nei due paesi hanno avuto un ruolo ancor più importante. In Francia, il cattolicesimo è la forza religiosa dominante se non addirittura egemonica. Ora, questa situazione pone dei problemi. Il cattolicesimo si oppone, infatti, alle grandi idee della modernità emergente. Portalis, il grande giurista di Napoleone I, sperava, al momento della redazione del Concordato (1801-1802), che la Chiesa avesse rinunciato alle sue nostalgie dell'Ancien Régime per entrare pienamente nel mondo dei Lumi. Ora, non è quello che è avvenuto. Man mano che avanza il XIX secolo, i fedeli di Roma si irrigidiscono in un' intransigenza sempre più accentuata, cui il Syllabus de 1864 offre una chiara illustrazione, condannando la proposta secondo la quale "il pontefice romano può riconciliarsi con la libertà, il progresso, la civiltà nuova " e coltivando il progetto di riportare ogni legge sotto il dominio della norma divina. Gli Stati Uniti presentano, per quanto li riguarda una tutt'altra configurazione. Qui è il protestantesimo, scisso in tante denominazioni, che domina la scena religiosa, e la cui tendenza è, al contrario dalla tendenza seguita dal cattolicesimo, di accompagnare la modernità, al tal punto che tanti dei suoi leaders non esitano, seguendo le elaborazioni di Roger Williams nello Stato di Rhode Island nel XVII secolo, a difendere persino l'idea stessa di separazione ${ }^{5}$.

Ecco ciò che ci riporta alla tipologia di Luca Diotallevi. In Francia, le condizioni erano favorevoli all'attuazione di un "separatismo assertivo". È specificamente in un tempo certo, nei primi anni del XIX secolo, che si è pensato di poter associare l'idea di Stato forte a quella di

5Su questi punti, David Martin, Religion and Power, No Logos without Mythos, London, Ashgate, 2014. Voir aussi Philippe Portier, " Modernités plurielles ? Une analyse longitudinale des régimes de régulation du croire dans les démocraties stabilisées ", in Micheline Milot, Philippe Portier et Jean-Paul Willaime (dir.), Pluralisme religieux et citoyenneté Rennes, PUR, 2009, p.220-255.

6

6 Ahmet Kuru, Secularism and State Policies toward Religion: The United States, France, and Turkey, Cambridge, Cambridge University Press, 2009. 


\section{L'ARTICOLAZIONE DEL POLITICO E DEL RELIGIOSO NELLE DEMOCRAZIE CONTEMPORANEE. UN DIALOGO CON LUCA DIOTALLEVI 7}

religione pubblica. Era la scommessa dei promotori del patto concordatario. L'arroccamento della Chiesa su un tradizionalismo ultramontano ha impedito che questo tipo di confessionalismo moderno, che è all'opera ancora oggi in certi paesi scandinavi ${ }^{7}$, potesse perseverare nell'essere. Il caso americano, organizzato intorno a un "separatismo passivo", proviene da un tutt'altro contesto. Se i significati religiosi ${ }^{9}$ hanno potuto mescolarsi nel cuore stesso dello spazio pubblico, con i significati politici, è perché lo Stato, sprovvisto di un qualsiasi progetto magisteriale, non si è trovato davanti ad una religione che contestasse la sua autonomia. Rimane ancora una questione non risolta: perché gli Stati Uniti hanno voluto proibire "l'istituzionalizzazione", come è stato il caso in altri paesi tali l'Inghilterra, favorevoli anch' essi all'ethos religioso? Si trattava precisamente qui, in un contesto segnato da un vasto pluralismo religioso, di permettere agli individui credenti di poter coltivare la propria appartenenza religiosa, senza essere né costretti, né influenzati, dalla scelta dello Stato in favore di una particolare religione. Sono dunque, all'origine, due modelli di separazione che si oppongono: in Francia la separazione è concepita nell'intento di liberare lo Stato dal potere del religioso, considerato come potenzialmente liberticidio; negli Stati Uniti, la separazione si è costruita nella prospettiva di permettere alla società civile la più larga possibile espressione del religioso, percepito qui come, per essenza, civilizzatore.

La seconda questione, più centrale, riguarda la trasformazione dei modelli di regolazione del credere. Il testo di Luca Diotallevi tende a fissarli in una stabilità antistorica. Ora, la storia ci confronta a schemi mutevoli. Gli Stati Uniti, per esempio, hanno conosciuto una svolta potentemente "secolarista" negli anni 1960-1970, sotto l'effetto di un intervento rinforzato dello Stato impegnato a partire dal New Deal, ma anche a causa di un processo di liberalizzazione dell'opinione pubblica. La Corte suprema ha prodotto in questa epoca una giurisprudenza che ha segnato più nettamente la separazione tra il politico e il religioso. Alcune sentenze hanno vietato i momenti obbligatori di preghiera all'interno della scuola, come nel 1962 la decisione Engel vs

7

In un contesto religiosamente diverso, dove la religione dominante è un luteranismo molto adattabile alla modernità.

8

Ahmet Kuru, ibid.

9

Non dimentichiamoci di ricordare che, se questo sistema accetta che delle scelte individuali si manifestino nello spazio dello Stato o l' espressione di una religione diffusa, è proibito qualsiasi riconoscimento ufficiale di una particolare religione.. 
Vitale, confermata, nel 2000, dalla decisione Santa Fe Independent School District vs Doe. La sentenza Lemon vs Kurtzman, nel 1971, per quanto la riguarda, si oppone, servendosi degli argomenti della sentenza Everson vs Board of Education del 1947, a ogni possibilità di finanziare le attività scolastiche confessionali. Ciò che ha permesso a una giurista francese, Elizabeth Zoller di dire che "gli Stati Uniti, hanno istituito un sistema altrettanto esigente come quello francese"10 .

È tuttavia vero, seguendo Diotallevi, che il periodo recente, sotto la pressione della desecolarizzazione descritta da Peter Berger, ha, per certi aspetti (pensiamo ai Faith Based Initiatives difesi da Bush e da Obama) ripristinato lo spirito "filoclericale" del periodo precedente. La Francia si è evoluta allo stesso modo, con la sua adesione, negli anni 1970, ai meccanismi dello Stato di diritto (con il rafforzamento del potere giuridico) esso stesso legato d'altronde a una rimessa in questione del repubblicanesimo dall'alto. Ormai lo Stato finanzia i culti, affida delle missioni di mediazione e perizie alle Chiese, apre le sue scuole all'insegnamento del fatto religioso, ammette nei cimiteri municipali degli spazi confessionali, così che si può sicuramente far rientrare il modello francese nella categoria del "separatismo moderato".

Che pensare di questa categoria, nella quale i commentatori inglobano la maggior parte degli Stati? Per Luca Diotallevi, questa categoria è decisamente troppo vasta. Non si tiene conto del fatto, spiega, che gli Stati che l'hanno istituita non hanno la stessa public agency, neanche la stessa relazione con la società civile (l'autore oppone, da questo punto di vista, il modello "corporativo" e il modello "associativo"). Il sociologo italiano ha in parte ragione. La Germania, per esempio, con il suo sistema di Stato sussidiario non è l'Inghilterra, con il suo regime di Stato liberale. Allo stesso tempo, si manifestano punti di convergenza: la loro iscrizione in un contesto similare, segnato dalla immigrazione, dalla perdita di potere dello Stato, dall'affermazione di rivendicazioni religiose, i regimi occidentali hanno tutti attuato, senza abolire tutti i tratti del loro sistema precedente, uno schema di pluralismo regolato (o di " neocorporativismo") fondato su interazioni più pesanti tra lo Stato e le Chiese. Indubbiamente è necessario uscire dal nostro "nazionalismo ideologico" e convenire che la seconda modernità, con l'entrata nell'universo della mondializzazione, ha in Occidente un effetto dissolvente sui modelli di secolarità nati dalla prima modernità ${ }^{11}$.

10

Elizabeth Zoller, La Laïcité américaine, Paris, Dalloz, 2005, p. 17.

1111 Philippe Portier, "Les laïcités à l'épreuve de la deuxième modernité ", in Jean Baubérot, Micheline Milot, Philippe Portier (dir.), Laïcité, Laïcités, Paris, Editions de la Maison des Sciences de l’Homme, 2014. 